\title{
Research on Recovery Strategy and Motion Control for Autonomous Underwater Vehicle
}

\author{
Yushan Sun, Lifeng Wang, Xiangrui Ran and Guocheng Zhang* \\ Science and Technology on Underwater Vehicle Laboratory, Harbin Engineering University, Harbin 150001, China \\ ${ }^{*}$ Corresponding author
}

\begin{abstract}
During the recovery of AUV, the hydrodynamic power of the hull becomes intricate due to the presence of the submarine. It's unable to accurately obtain the forces and moments in all degrees of freedom. In this paper, we put forward the plan of traversing, depth control and path planning for complex hydrodynamic environment, and propose a method of optimizing $S$-plane motion control parameters based on improved simulated annealing algorithm. The simulated annealing algorithm is improved by selecting the initial temperature, annealing algorithm, increasing the memory function, stopping the temperature setting and so on. Through using this algorithm to optimize the $S$-plane controller parameters, the automatic adjustment of control parameters can be realized. The simulation results show that the improved method is superior to the ordinary $S$-plane control method, with better control accuracy and faster response. The recovery process achieves the desired result.
\end{abstract}

Keywords-AUV recovery; Motion control; S plane; Simulated annealing algorithm

\section{INTRODUCTION}

The 21st century is the century of the ocean. Autonomous underwater vehicles are gradually becoming an important means for mankind to explore and exploit the marine resources. Its field of application has been expanding and has been widely used in scientific investigation, underwater engineering, marine research and development of marine resources [1-2]. Intelligent underwater vehicle is the future trend of development. AUV is capable of autonomous decision-making in the execution of its mission. It has great advantages that its range of activities is not restricted by cables. However, when it comes with limited energy resources and communication difficulties with the outside world, it must often return to its mother ship for energy supply and information exchange. Therefore, placing and recovery near work areas is an unavoidable task for AUV applications. Deploying and recovering AUVs, however, is currently largely limited to surface vessels or swimmers [3]. It is attracting the attention of AUV researchers around the world that how to recover the AUV safely and conveniently after it finishes its mission or receives a return instruction, which is also an important part of intelligent underwater robot research .

Recovery of underwater vehicles has always been a more difficult problem, especially the recovery of the AUV, which is constrained by many factors, such as the precise positioning of recovery areas, the recovery point determination, precise control in complex sea conditions [5]. Subjectively, many problems such as planning control and fluid dynamics that need to be solved in recovery run through the entire robot research. It not only brings about the expansion and improvement of other researches such as path planning, localization and simulation of sonar, precise control and optimization and improvement of hydrodynamic model, but also is an important manifestation of the overall research strength of AUV. However, up till now, no breakthrough has been made on this issue, so it needs further exploration and research.

The hydrodynamic system of AUV is non-linear and timevarying. The hydrodynamic coefficients are often difficult to be accurately estimated or measured by the test. It is impossible and unrealistic to establish an accurate AUV motion model. Xuemin LIU, Yuru XU and others derived Splane control which is simple and effective, by drawing on the fuzzy logic control and drawing on PID control methods [5]. Jiancheng LIU advances sub-S plane control, which is based on the approach shown in literature [6], increases the integral part of the controller, and ext racts Sig moid function. However, the above method does not have self-learning ability, and the control parameters still need manual adjustment. The simulated annealing algorithm can effectively avoid falling into local minimum and eventually tend to global optimum by giving a kind of probabilistic jumping property which is time-varying and eventually tends to zero to the search process. In this paper, the parameters of the controller are automatically optimized by simulated annealing algorith $\mathrm{m}$ to improve the performance of the AUV's motion control and enhance its robustness.

\section{RECOVERY STRATEGY AND PATH PLANNING}

\section{A. Embedded Carring Recovery Scheme of AUV}

Embedded carrying recovery is used in AUV recovery program in this paper. Due to the special considerations and requirements of embedded carrying recovery, hydrodynamics becomes complicated and changeable because of the existence of submarines. It can be seen from the calculation of the hydrodynamic forces of the hull under the interference of submarines that due to the complexity of computational fluid dynamics in this special flow field, it is unrealistic and impossible to obtain exactly six degrees of freedom of force and moment. Therefore, so far, only one of the degrees of freedom of the hull can be obtained accurately. In this way, there are special requirements for the recovery control scheme: the hull can only be controlled slowly toward the submarine in the direction of one degree of freedom. To this end, this article set up two kinds of recovery program: recovery by traverse 
control and depth control. The entire recovery process is as follows:

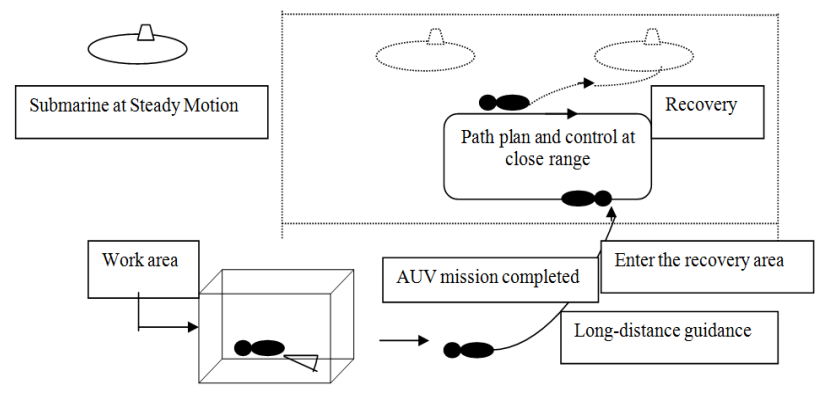

FIGURE I. RECOVERY PROCESS

Recovery by traverse control refers to that when AUV enters the recovery area, slowly approaching the submarine podium from the side of submarine, and finally reaching the recovery target location. The details of the scheme for AUV recovery by traverse control are as follows:

That is to recovery AUV at close range from the side of submarine. First of all, it takes remote control far in front of the submarine regard less of the impact of submarine, which is equivalent to direct motion to the middle switching area. In this phrase, the AUV is about $10 \mathrm{~m}$ away from the submarine centerline in the lateral direction and $6 \mathrm{~m}$ away in the depth direction, which means the lateral distance and the depth are constant. When the AUV reaches the middle switching area, it switches from remote control to close and precise control because it is necessary to consider the effect of the submarine which leads to hydrodynamic changes. At this moment, the AUV moves laterally and slowly toward the target point. It considers dynamic positioning after reaching the target point, and if the control accuracy meets the requirements, the dynamic positioning can be neglected. The hull should be kept a small float within the error range before the subsequent recovery work.

Recovery by depth control refers to that it takes long distance control far from the submarine. The AUV can sail from all directions on the side of the submarine to the middle switching area, and switch to close and precise control. The situation is similar to recovery by traverse control.

\section{B. Path Planning for Recovery Process}

In the actual recovery process, there are two points that need to be focused and planned. The first is the switching area from long-range to short-range recovery, which requires common plan of geometric paths and hull attitude. The next is the path planning of the AUV in the recovery area, which belongs to the category of local path planning. In order to avoid the collision between the AUV and the submarine, collision avoidance logic should be added to path planning.

\section{1) Path Planning of the Switcning Area}

The path planning of the switching area can be described in detail as follows: In the critical area within the submarine's range of action to the AUV in the switching area, the hydrodynamic power of the hull becomes intricate and difficult to control due to the presence of the submarine and its podium. To accurately calculate the force and moment in the direction of six degrees of freedom is unrealistic, and it can only be simplified as considering the force in a certain direction alone, which requires the hull to maintain a suitable attitude into the recovery area. Due to the special requirements of control accuracy of the recovery operation, it is necessary to ensure that the AUV keeps a low speed near zero when entering the recovery area in order to accomplish the AUV recovery by close and precise control in such a complicated flow field. In order to meet the above two conditions prepare well for AUV recovery by traverse control, this paper designs the following path planning scheme:

(1) In order to ensure that the AUV maintains a low speed in the switching area, an intermediate target point needs to be set in the switching area. The entire recovery process is divided into two phases: the movement of long-range to the intermediate target point and the precise control at close range in the recovery area.

(2) In order to ensure the AUV and submarine centerline parallel to each other, the AUV has to make fixed-point motion in the switching area. To this end, the plane area is divided into the following sections for discussion, as shown:

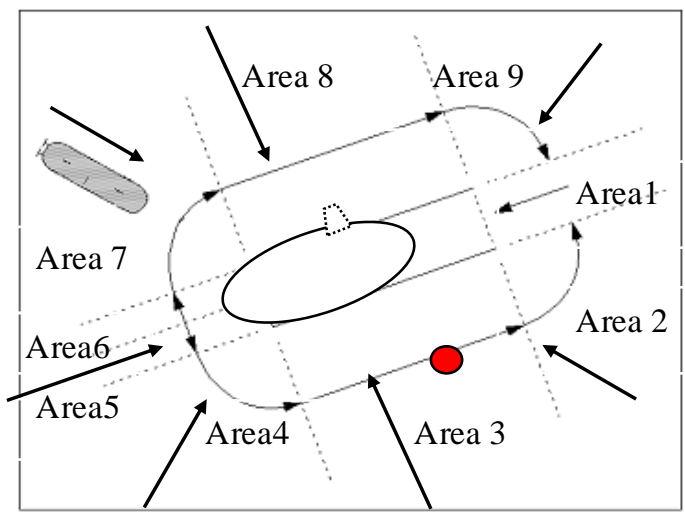

FIGURE II. THE SWITCHING AREA

Analysis: As shown in the figure, the switching area can be divided into nine areas shown in the XY two-dimensional plane. Since the two-dimensional plane where the submarine is located is symmetrical about the $\mathrm{X}$ and $\mathrm{Y}$ axes, it is only possible to consider the case of the area 1 to the area 3.

For area 1, the AUV is somewhere just in front of the submarine. Given the complexity of interference caused by the head of submarine on the AUV flow field, it is impossible to recovery directly from the bow portion. The path planning scheme must ensure that the AUV moving outside the critical area from the initial position to the switching point. When the AUV arrives at the switching point, it is ensured that the centerlines of the AUV and submarine parallel to each other, and gets ready for recovery at close range.

For area 2, similar to area 1 , the difference is that the AUV's initial position is not just in front of the submarine, but closer to the middle switching point and easier.

For area 3 , it is equivalent to the direct motion to the middle point. Fixed-point control will be taken after reaching the switching point. 


\section{2) Path Planning of the Recovery Area}

AUV path planning of the recovery area belongs to the category of local path planning. In order to avoid the collision between AUV and submarine or other obstacles in the recovery area, path planning of collision avoidance logic should be added. The short baseline sonar can only get the approximate position of the podium, so the AUV that receives the recovery instruction can only reach the area near the podium without being accurately retrieved to the desired location on either side of the podium. At th is point, careful planning and precise controls are required to ensure that the desired location is found. The recovery operation needs to be successfully completed and guaranteed that there is no collision with the submarine podium. In this way, path planning becomes a very prominent issue in recovery operations. In recognizing the desired position of the podium, the AUV needs to integrate various aspects of the data information through the understanding of its location and environment so as to obtain the exact information of the desired location and plan an appropriate route. The entire planning process in the recovery area involves two issues:

- A comprehensive understanding of sensor information and environmental information (recovery beacons placed on submarine podiums, etc.);

- Add collision avoidance logic and plan the right path according to the information obtained.

\section{THE IMPROVED S PLANE CONTROLLER}

\section{A. S Plane Control}

S-plane control is based on the fuzzy control, which goes on nonlinear fitting for the table of control rules. As shown in Figure III, the Sig moid surface is used instead of the fuzzy rule base, thereby obtaining the S-surface control[7].

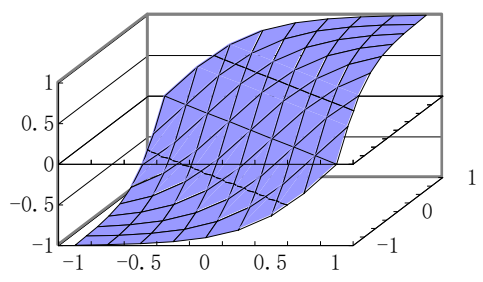

\section{FIGURE III. SIGMOID FUNCTION CURVED SURFACE}

The Sigmoid surface function is

$$
z=2.0 /\left(1.0+e^{\left(-k_{1} x-k_{2} y\right)}\right)-1.0
$$

Therefore, the motion control model of AUV recovery is

$$
u=2.0 /\left(1.0+e^{\left(-k_{1} e-k_{2} \&\right)}\right)-1.0+\Delta u
$$

In the formula, $e$ and \&are deviation and rate of deviation which are the input information of control system. $u$ is the output, which means the output force on each degree of freedom. $k_{1}$ and $k_{2}$ are the control parameters corresponding to the deviation and rate of deviation. $\Delta u$ indicates a fixed disturbance to the AUV. The adaptive approach is as follows:

a) Judge whether the speed of the AUV is less than a set threshold, if yes, switch to b), otherwise, switch to c);

b) Assign the deviation of the degree of freedom to a set array and add 1 to the set count value. When the count value reaches a predetermined value, switch to d);

c) Set the value of the array one channel forward, meanwhile minus lof the value, switch to a);

d) Get the weighted average of the value of the array. The average deviation is used to calculate the offset of the AUV's motion control output. Adaptively adjust controller output to eliminate fixed control deviation, while set the array and count value to zero, execute the next cycle.

Although there are fewer parameters needed to be adjusted of S-plane controller in the motion control of AUV recovery, these parameters need to be adjusted manually. The improper parameter setting will seriously affect the control precision of the underwater vehicles. To solve this problem, we introduce the simulated annealing algorithm to optimize the relevant parameters of the controller automatically.

\section{B. The Improved SA Algorithm}

Simulated Annealing Algorithm (SA algorithm) was first proposed by Metropolis and others. In 1983, Kirkpatrick applied it onto combinatorial optimization. SA algorithm is a random optimization algorithm based on Mente Carlo's iteration solution strategy. The origin of SA algorithm is the physical annealing process of solid substances, which is similar with a general combinatorial optimization problem [89].

The initial temperature and the way to reduce the temperature are the key to the SA algorithm, which affect the overall performance and the convergence of algorith $\mathrm{m}$. When the initial temperature is high or the temperature drops slowly, the search process becomes slow and takes up a lot of resources. When the initial temperature is low or the temperature drops quickly, it is difficult to find the global optimal solution the search process becomes too fast. Considering the above problems, we improve the simulated annealing algorithm.

The process of the improved SA algorithm is as follows:

1) Selection of initial parameters: Select initial state $S$ of the AUV randomly, and set the initial temperature as $T_{0}=k \gamma$, $\gamma=\max \left\{g_{j} j \in D\right\}-\min \left\{g_{j} \mid j \in D\right\}$. Determine the value of $k$ through experiments. Determine the number of iterations $L_{0}$, and set $L_{0}=\beta \cdot L_{0}, \beta \geq 1$. Initialize the number of iterations $L=$ 0.

2) Generate a random new state $S^{\prime}=S+\operatorname{Rand}(0,1) \cdot \delta$, here, $\delta$ is a given step size, $\operatorname{Rand}(0,1)$ is a random number between 0 and 1 . Calculate the difference between performance index $\Delta=g\left(S^{\prime}\right)-g(S)$. 
3) If $\Delta<0$, make $S=S^{\prime}$, then switch to 4), otherwise, accept $S^{\prime}$ in the probability of $\exp (-\Delta / T)$. If $\xi<\exp (-\Delta / T)$, $\xi$ is a random number between 0 and 1 , set $S=S^{\prime}$, otherwise $S$ is unchanged.

4) $L=L+1$. If $L<L_{0}$, return to 2), otherwise switch to 5).

5) Reduce the temperature. Set $T_{i+1}=\alpha T_{i}$, here, $\alpha$ is attenuation coefficient, $\eta$ is adjustment factor. Set $\alpha=\alpha+\eta$, $0 \leq \eta<1,0<\alpha<1$. Increase the number of iterations at each temperature.

6) Determine whether the annealing process is over according to the following conditions. (1)The total number of cycles reached a predetermined value; (2) $T$ is less than a certain threshold; (3)There is no change in the value of the two successive calculations of $g(S)$. (4) The current error is less than the prescribed error. When any one of the above conditions is met, switch to 7), otherwise, switch to 2).

7) Output the current $S$ as the optimal solution and end.

In order to ensure that the final solution of the algorith $\mathrm{m}$ is the best in the whole search process, we introduce memory function into the algorithm. Set variables A and B to store the current optimal state and the performance index in the program. Only when the new performance index $g\left(S^{\prime}\right)<g^{*}$, set $S^{*}=S^{\prime}$ and $g^{*}=g\left(S^{\prime}\right)$. When the annealing process is finished, this method can ensure that the solution is the best solution in the whole period.

\section{Result of Simulation Experiment}

For AUV state optimization calculation of performance indicators, we use ITAE integral the performance index:

$$
g(I T A E)=\int_{0}^{\infty} t \mid e(t) d t=\min
$$

In order to facilitate the simulation experiment, we discretize the above formula:

$$
g=\sum_{k=0}^{\infty} t_{k}\left|e\left(t_{k}\right)\right| T=\min
$$

Parameter settings: The initial temperature $T_{0}=k \gamma, k=75$. Initial decline coefficient $\alpha=0.8, \alpha \leq 0.95, \mu=0.07$. Iterate time increase coefficient $\beta=1.005$.

Taking the heading angle control as an example, in the integrated simulation environment, the response curve of the $\mathrm{S}$ plane control and the improved control is shown in FIGURE IV. As can be seen from the simulation results, the improved $S$ plane control can increase the convergence rate and get better control effect. The response curve of north position and depth control of the AUV is shown in FIGURE V. The control system based on the improved S plane control has high control accuracy and stability. It can meet the requirements of motion control in AUV recovery.

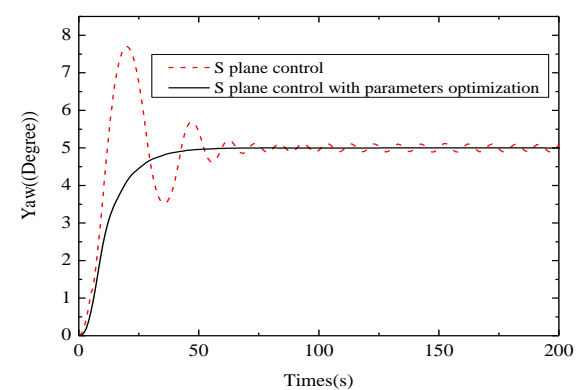

(a) initial parameters: $k_{1}=9.0, k_{2}=8.0$

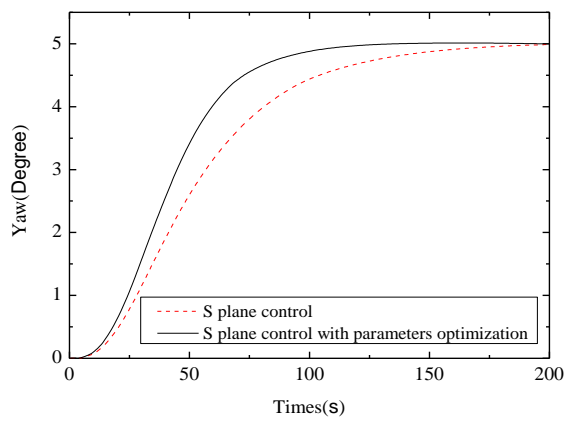

(b) initial parameters: $k_{1}=1.5, k_{2}=1.2$

FIGURE IV. SIMULATION RESULTS OF AUV

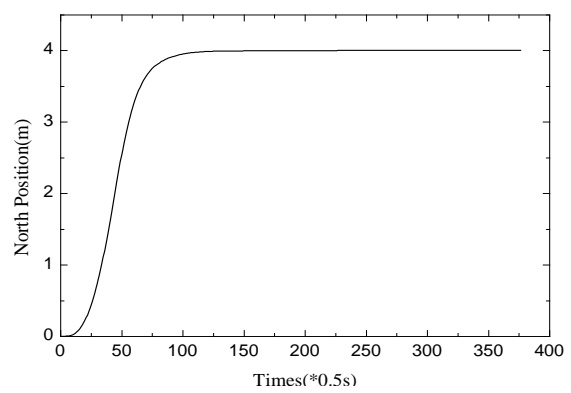

(a) Results of north position control

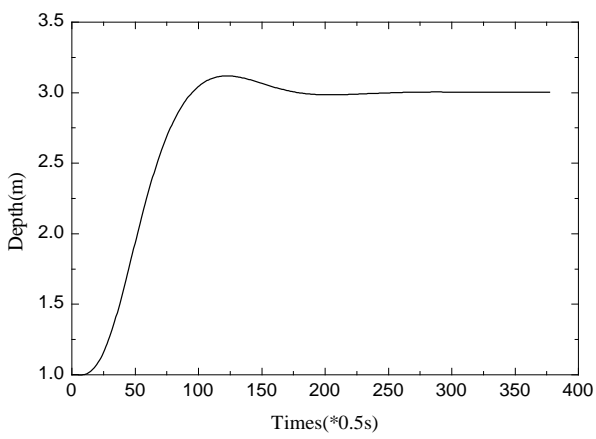

(b) Results of depth control

FIGURE V. SIMULATION RESULT S FOR POSITION CONTROL

\section{SIMULATION OF AUV RECOVERY}

According to the working way of AUV, the simulation of motion control is carried out on the established visual simulation platform. Long-range guided navigation, dynamic positioning at close range and docking process are as follows: 


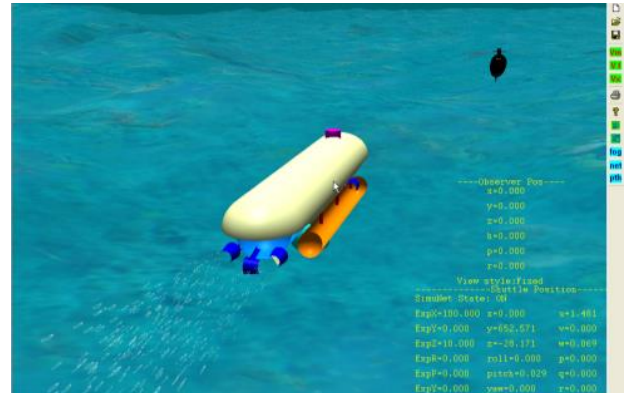

(a)Guide AUV to submarine through aquatic communication

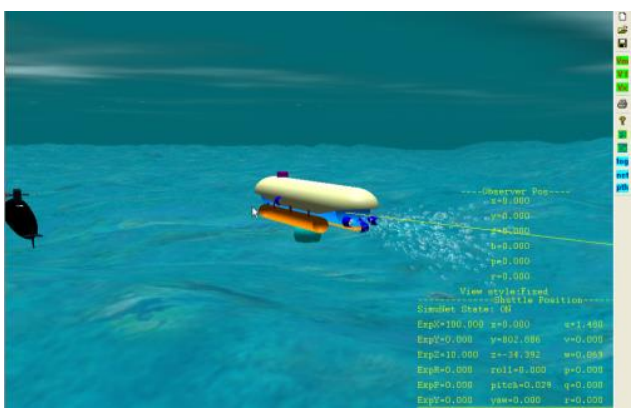

(b) Enter the range of the short baseline sonar

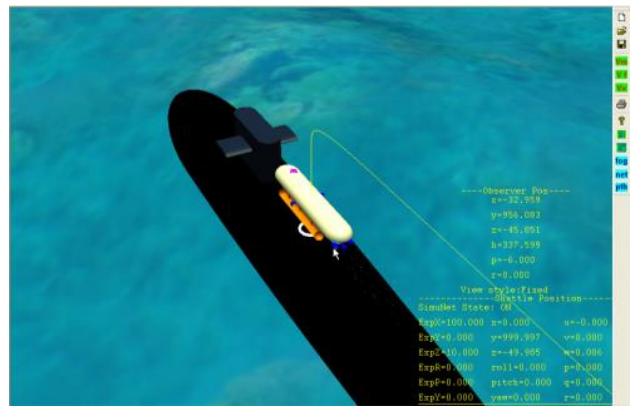

(c) Dynamic positioning at short range

FIGURE VI. SIMULATION OF AUV RECOVERY

\section{CONCLUSION}

Force and moment can be accurately got in only one direction because of the submarine interference in the underwater recovery process. For recovery control, the Splane controller is a simple and practical control method with few parameters during the control of recovery, but the parameters need to be manually adjusted. In this paper, recovery program based on traverse, depth control, as well as path planning scheme for AUV recovery are designed. In addition, an improved S-plane controller based on the improved simulated annealing algorith $\mathrm{m}$ is proposed for AUV recovery control. The simulation results show that the improved control algorith $\mathrm{m}$ is more efficient and faster than the conventional S-plane control algorith $\mathrm{m}$. It is applied to the motion control of AUV recovery, which has good stability and achieves the desired results.

\section{ACKNOWLEDGMENT}

This research was part of the autonomous underwater vehicle scientific research project (Grant No.51779057, 51709061) supported by National Natural Science Fund of
China. It has also been partially supported by the pre-feasibility study project (Grant No.41412030201) and the project (Grant No.2015ZX01041101) supported by Major National Science and Technology Project. Great thanks are addressed to them by the research team.

\section{REFERENCES}

[1] Xu Yuru, Li Pengchao, "Devepoling Tendency of Autonomous Underwater Vehicles," Chinese Journal of Nature, 2011, 33(3):125132.

[2] Xu Yuru, Pang Yongjie, Gan Yong, Sun Yushan. "AUV—state-of-the art and prospect", CAAI Transactions on Intelligent Systems, 2006,1(1):9-16

[3] D. Chapuis, C. Deltheil and D. Leandre. Determination and Influence of the Main Parameters for the Launch and Recovery of an Unmanned Underwater Vehicle From a Submarine. Proceedings of the 1996 Symposium on Aut onomous Underwater Vehicle Technology, Monterey California, June 1996: 276-282P

[4] Yakimenko O A, Horner D P, Pratt D G. "AUV rendezvous trajectories generation for underwater recovery," Control and Automation, 2008, Mediterranean Conference on. IEEE, 2008:1 192-1 197

[5] Liu Xuemin, Xu Yuru. "S control of automatic underwater vehicles", Ocean Engineering, 2001,19(3): 81-84

[6] Liu Jiancheng, Yu Huanan,Xu Yuru. "ImprovedS plane control algorithm for underwater vehicles," Journal of Harbin Engineering University,2002, 23(1):33-3

[7] Li Yueming, Pang Yongjie, and Wan Lei. "Self-Adaptive S-Plane Control for Underwater Vehicles." Journal of Shanghai Jiaotong University V46.2 (2012): 195-200.

[8] ZHANG Hao, TAO Ran, LI Zhi-yong, et al. A feature selection method based on adaptive simulated annealing genetic algorithm. Acta Armamentarii, 2009,30(1): 81-85.

[9] Wang Ling. "Intelligent Optimization Algorithm"[M], Beijing: T singhua University Publishing Company, 2001 